\title{
El uso del potencial dialógico de Twitter: el caso de las marcas de moda
}

institucional.us.es/ambitos/

\section{Marián Navarro-Beltrá}

Universidad Católica de Murcia

mnavarro2@ucam.edu

\author{
Susana Miquel-Segarra \\ Universitat Jaume I de Castellón \\ smiquel@uji.es

\section{Irene García Medina} \\ Glasgow Caledonian University \\ irene.garcia2@gcu.ac.uk
}

English Version: The use of Twitter's dialogical potential: the case of the fashion brands.

\section{Resumen}

Las marcas de moda pueden ver en el uso de las redes sociales una manera de interactuar con sus stakeholders que puede mejorar las relaciones que mantienen con ellos y su imagen. Así, se pretende analizar la comunicación que las marcas de moda realizan a través de Twitter. Para ello, se ha realizado un análisis de contenido de 1135 tweets publicados en 2016 por H\&M, Zara, Ralph Lauren y Hugo Boss.

Aunque no existe consenso entre las marcas estudiadas se puede afirmar que, en general, Twitter posee un amplio potencial dialógico que las marcas de moda no están aprovechando plenamente.

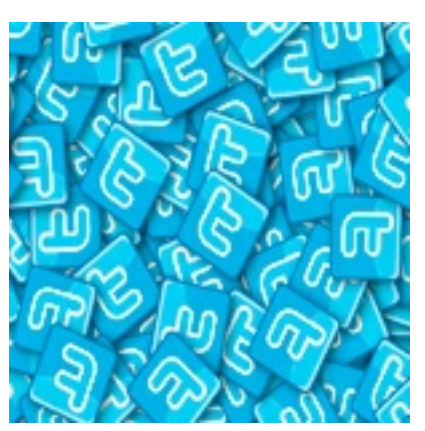

\section{Palabras clave}

Twitter, redes sociales, moda, comunicación corporativa, diálogo.

Fashion brands use social networks astract them and their image. Thus, in this study, the communication that fashion brands made through Twitter is analyzed. In order to do this, a content analysis of 1135 tweets published in 2016 by H\&M, Zara, Ralph Lauren and Hugo Boss has been carried out. Although there is no consensus among the studied brands, we can say that in general, Twitter has a large potential for dialog with its customers that fashion brands are not fully exploiting yet.

Keywords

Twitter, social networks, fashion, corporate communication, dialogue.

\section{INTRODUCCIÓN}

Las organizaciones observan como cada día más las redes sociales e Internet influyen en la imagen que se tiene de ellas. Los patrones tradicionales de la comunicación se han adaptado a un nuevo contexto que se encuentra influenciado por las tendencias que marcan las redes

ÁMBITOS
2017
n-35
sociales (Atarama y Cortéz, 2015: 27). Para obtener y mantener la reputación es imprescindible establecer un vínculo de alto nivel relacional con los grupos de interés (Villafañe, 2004: 193) y en este sentido "el diálogo se ha establecido como una acción necesaria para generar un vínculo de valor entre una institución y su público" (Atarama y Cortéz, 2015: 29). De esta forma, la bidireccionalidad es clave para generar confianza y 
actualmente en las redes sociales podemos encontrar esta posibilidad, ya que la conversación se ha convertido en la esencia de la nueva era de Internet (Villanueva, Aced, Armelini, 2007: 3).

Entre las redes sociales existentes Twitter llama especialmente la atención, ya que el libre acceso a la información y la brevedad de sus mensajes lo convierte en un canal idóneo para controlar y gestionar la comunicación reputacional de las organizaciones (Atarama y Cortéz, 2015: 26). Así, la importancia de esta red social como herramienta de comunicación con sus públicos está empezando a llamar la atención de la academia y se está empezando a convertir en un tema frecuente de investigación (Alonso, 2016; Castelló, Del Pino y Ramos, 2014; Castelló y Ros, 2012; Guzmán, Del Moral, González y Gil, 2013; Simelio y Molina, 2014).

Por su parte, cabe señalar que la moda está estrechamente ligada a los medios sociales, situación que puede ser explicada al considerar a esta industria como la más dinámica que existe, ya que cada pocos meses surgen nuevas tendencias y estas son compartidas con amigos y familiares (Sharma y Shani, 2015: 503). De hecho, las marcas de moda cada vez tienen más importancia en este tipo de medios (Manikonda, Venkatesan, Kambhampati y Li, 2014), de forma que su uso es considerado como una potente herramienta que puede utilizar dicha industria para fomentar su comercialización (Manikonda, Venkatesan, Kambhampati y Li, 2014). Así, las marcas de moda pueden ver en el uso de las redes sociales una manera de interactuar con sus stakeholders de forma directa que puede mejorar las relaciones que mantienen con ellos e, incluso, su imagen.

Pese a la importancia que puede suponer el uso de Twitter para las marcas de moda como herramienta de comunicación con sus stakeholders, esta temática no ha sido analizada con frecuencia por parte de la academia. Ante esta escasez de investigaciones resulta de vital importancia estudiar cómo las marcas de moda se comunican con sus públicos a través de Twitter. Así, el aumento de la popularidad de esta herramienta y su capacidad para proporcionar amplias posibilidades de involucrar a sus públicos presentan una oportunidad para examinar el uso que las marcas de moda realizan de esta plataforma y analizar si fomentan la comunicación dialógica con sus stakeholders.

De esta forma, el objetivo del presente trabajo se basa en analizar la comunicación que las marcas de moda realizan a través de Twitter. En concreto, se pretende estudiar si existe consenso entre las marcas de moda con relación a la conservación de los visitantes, la generación de visitas reiteradas, la aportación de información útil y el mantenimiento del bucle dialógico.

\section{METODOLOGÍA}

Con el propósito de alcanzar los objetivos planteados anteriormente se ha recurrido a la metodología cuantitativa. Así, se ha realizado un análisis de contenido de todos los tweets publicados por H\&M, Zara, Ralph Lauren y Hugo Boss entre el 1 de enero y el 30 de junio de 2016, ya que la recogida de datos tuvo lugar en julio de ese mismo año y se pretendía obtener y analizar la información más reciente posible. Las marcas mencionadas anteriormente han sido escogidas por ser las cuatro únicas que aparecen en la categoría "ropa" del ranking The best global brands (2015) realizado por Interbrand (1).

Para la selección del perfil a analizar, en caso de que la marca tuviera más de una cuenta de Twitter, se escogió la internacional, es decir, se descartaron aquellas que eran específicas para determinados países. De esta forma, los perfiles examinados fueron: @hm, @ZARA, @RalphLauren y @HUGOBOSS. Todos ellos incluían el identificativo "cuenta verificada".

Con el propósito de obtener los tweets específicos a analizar se recurrió al programa informático Twitonomy. Tras seleccionar las fechas y los perfiles especificados anteriormente se obtuvo un total de 1135 tweets. La totalidad de estos tweets fueron codificados y examinados para la realización del presente estudio.

Para ello, se elaboró un protocolo de codificación formado por 47 variables que recogía información sobre los siguientes aspectos: características de la marca, texto, URL, particularidades del tweet, menciones, hashtags, enlaces, retweets, favoritos, preguntas y comentarios efectuados por la marca y por sus seguidores. 
Con el propósito de que el protocolo de codificación tuviese un alto grado de fiabilidad, se siguieron las recomendaciones establecidas por Wimmer y Dominick (1996: 184-185) que consisten en definir al máximo los límites de las categorías, adiestrar a los codificadores y realizar un ensayo previo.

Así, para la realización de esta investigación, el protocolo de codificación se acompañó de un manual de instrucciones. Además, con la finalidad de poner en práctica estos documentos y de aprender a utilizarlos correctamente, se realizaron diversas sesiones prácticas. En estas sesiones, las posibles discrepancias encontradas en la codificación de las variables fueron resueltas a través de la discusión y el consenso por parte de las personas implicadas en el proceso de codificación. Posteriormente se realizó un ensayo previo con resultados satisfactorios y, a continuación, se calculó la fiabilidad intercodificadores. Para ello, y con anterioridad a realizar la codificación definitiva, los investigadores implicados analizaron de manera independiente una misma submuestra del 15\% de los tweets a estudiar, es decir 170, y obtuvieron un índice medio de Kappa de Cohen de 0,974 (2).

Con referencia a la validez, cabe señalar que las variables utilizadas en el protocolo de codificación son el resultado de adaptar anteriores investigaciones. En concreto, el presente estudio parte de los principios dialógicos aplicables a Internet establecidos por Kent y Taylor (1998) y adaptados posteriormente para Twitter por Rybalko y Seltzer (2010). De esta forma, los principios dialógicos analizados en este trabajo son:

1. Aportación de información útil para los públicos: este principio hace referencia a la necesidad que posee la organización de ofrecer información de valor a sus stakeholders, ya que esta es la base para mantener una relación dialógica, pues los públicos confían en instituciones que proporcionan información útil y confiable (Kent y Taylor, 1998: 327-328). Al tener en cuenta la brevedad de las publicaciones realizadas a través de Twitter, se ha considerado que la forma más adecuada de incluir información útil es a través de enlaces. Por ello, se han analizado los enlaces y los lugares a los que remiten (imágenes, videos, música, páginas web y medios sociales, todos ellos de interés para los clientes de marcas moda).

2. Conservación de los visitantes: este apartado se basa en el peligro de incluir links que favorezcan que los seguidores naveguen por espacios no relacionados con la organización (Kent y Taylor, 1998: 330-331). De esta forma, se ha considerado como una presencia ampliada y adecuada de la propia institución la existencia de enlaces corporativos, aquellos que redirigen a otros sitios webs gestionados por la propia marca (Rybalko y Seltzer, 2010: 338). Además, para intentar mantener a los visitantes en la red social seleccionada es importante la publicación regular de tweets (Rybalko y Seltzer, 2010: 338).

3. Generación de visitas reiteradas: este punto hace referencia a la necesidad que posee la institución de incluir información atractiva para sus públicos capaz de fomentar que estos les vuelvan a visitar (Kent y Taylor, 1998: 329). En este sentido, como Taylor, Kent y White (2001: 270) sugieren, para construir relaciones se necesita tiempo y confianza, aspectos que únicamente se pueden conseguir a través de interacciones repetidas. Así, y siguiendo las indicaciones de Rybalko y Seltzer (2010: 338), los siguientes enlaces fomentan las visitas reiteradas: lugares corporativos donde se puede solicitar información adicional, espacios que describen eventos de la empresa, noticias propias en medios de comunicación, foros de discusión y sitios en los que se resuelven preguntas frecuentes -Frequently Asked Questions (FAQs)-. A estos enlaces se ha añadido el de la venta on-line, por la importancia que supone para las marcas de moda. Además, se ha considerado oportuno analizar el uso de hashtags y de menciones en el texto del tweet, ya que estos indicadores favorecen el engagement y potencian los vínculos entre la marca y sus clientes.

4. Mantenimiento del bucle dialógico: este principio se basa en la posibilidad que poseen los seguidores de realizar preguntas a la organización y la oportunidad que esta tiene de responder a las dudas, a las preocupaciones y a los problemas de sus públicos (Kent y Taylor, 1998: 326-327). Por tanto, en este apartado se analiza si las organizaciones conversan con sus stakeholders al plantear preguntas en sus tweets o al responder a comentarios o cuestiones realizadas por sus seguidores (Rybalko y Seltzer, 2010: 338), textos que también son examinadas. Además, se ha incluido el análisis de los retweets y de los "me gusta" (favorito) que obtienen las marcas en cada una de sus publicaciones, ya que estos indicadores suponen una interacción directa con el tweet por parte de los públicos y colaboran en la difusión del mensaje. 
Finalmente, y con el propósito de examinar la información recolectada, se utilizó el programa informático SPSS. En concreto, se recurrió a los descriptivos, a las frecuencias, a las tablas de respuesta múltiple, a los gráficos de líneas y al test Chi-cuadrado de Pearson (3).

\section{RESULTADOS}

\subsection{Características de los perfiles analizados}

Como podemos ver en la tabla 1, la cuenta de H\&M es la que mayor número de seguidores tiene (7.954.499), seguida a gran distancia de Ralph Lauren (1.841.305), Zara (1.149.376) y Hugo Boss (607.315). En los perfiles examinados, coincide la marca con mayor número de seguidores con aquella que primero inició su actividad en Twitter y con la que mayor número de tweets ha publicado en el periodo estudiado.

Si analizamos el ratio entre seguidores (followers) y aquellos a los que la marca sigue (following), que nos indica el índice de reciprocidad del seguimiento por parte de las marcas hacia sus seguidores, vemos como en ninguno de los casos supera el $0,033 \%$, siendo el de H\&M el más bajo $(0,004 \%)$.

Finalmente, si observamos la cantidad de tweets publicados por los perfiles, vemos como H\&M encabeza la clasificación, con un total de 9.385 mensajes y 404 durante el período de 6 meses analizados. Hugo Boss se sitúa en segundo lugar, con 6.043 y 346 respectivamente. Ralph Lauren, con 3.536 y 260 ocupa el tercer lugar, y Zara, está en el último puesto con 1.329 mensajes totales y 125 publicados durante el período de análisis.

Tabla 1. Características de los perfiles analizados

\begin{tabular}{|l|c|c|c|c|}
\hline & H\&M & Zara & Ralph Lauren & Hugo Boss \\
\hline Perfil & @hm & @ZARA & @RalphLauren & @HUGOBOSS \\
\hline Tweets & 9.835 & 1.329 & 3.536 & 6.043 \\
\hline Following & 293 & 71 & 384 & 203 \\
\hline Followers & 7.954 .499 & 1.149 .376 & 1.841 .305 & 607.315 \\
\hline Inició Twitter & Abril, 2008 & Agosto, 2011 & Abril, 2009 & Junio, 2010 \\
\hline Idioma & inglés & inglés & inglés & inglés \\
\hline $\begin{array}{l}\text { Tweets } \\
\text { analizados }\end{array}$ & 404 & 125 & 260 & 346 \\
\hline
\end{tabular}

Fuente: elaboración propia a través de Twitonomy. Informe generado el 11 de Julio de 2016

\subsection{Características de los tweets: idioma, plataforma utilizada y tipo de tweet}

Con referencia al idioma cabe destacar que la lengua inglesa es la utilizada principalmente por todas las marcas para redactar sus tweets. No obstante, se producen diferencias estadísticamente significativas con relación a esta cuestión $\left(x^{2}=132,913 ; p=0,000\right)$, ya que Zara es la única marca que recurre a otras lenguas. En concreto, de sus 125 tweets, 4 están escritos en español, 6 en francés, 2 en italiano, 2 en rumano y 2 en griego.

Las marcas también muestran desemejanzas estadísticamente significativas en función de la plataforma utilizada para publicar sus tweets $\left(x^{2}=1349,182 ; p=0,000\right)$. Así, aunque Ralph Lauren $(73,5 \%$ de sus publicaciones), Hugo Boss $(55,5 \%)$ y Zara $(51,22 \%)$ usan principalmente Twitter Web Client, H\&M se decanta por Adobe Social $(83,4 \%)$, plataforma que únicamente es utilizadas por esta marca. Además, cabe mencionar que Hootsuite solo es empleada por Hugo Boss (13,9\%) y Twitter Ads solo es usada por Ralph Lauren $(0,8 \%)$.

Si analizamos el tipo de tweet también apreciamos diferencias estadísticamente significativas en función de la marca $\left(x^{2}=190,557 ; p=0,000\right)$. De esta forma, a pesar de que todas se decanten por publicar sus propios 
tweets, Zara es la única que no utiliza ninguna otra tipología, ya que Ralph Lauren, Hugo Boss y H\&M recurren a los retweets ( $14,6 \%, 4,9 \%$ y $2,5 \%$ de las ocasiones respectivamente). De forma singular destacamos que $\mathrm{H} \& M$ es la única marca que también recurre a las respuestas $(18,1 \%)$, tipología de mensaje que lleva implícita la interacción directa con los seguidores

\subsection{Principio de aportación de información útil para los públicos}

Aunque es habitual que las marcas incluyan enlaces en sus tweets, Zara es la única que los incorpora en todas sus publicaciones. Así, Hugo Boss lo hace en el 99,7\% de los casos, H\&M en el $97 \%$ y Ralph Lauren en el $92,7 \%$. Estas diferencias son estadísticamente significativas $\left(x^{2}=30,862 ; p=0,000\right)$.

También se producen este tipo de desemejanzas al considerar el número de enlaces que contiene cada tweet en función de la marca analizada $\left(x^{2}=403,777 ; p=0,000\right)$. De esta forma, Ralph Lauren y Hugo Boss suelen incluir únicamente un enlace $(81,9 \%$ y $85,8 \%$ de los casos respectivamente), mientras que Zara prefiere incorporar dos (93,6\%). Por su parte, H\&M es la única que ha llegado a introducir tres enlaces en un mismo tweet, aunque solo en una ocasión.

Cuando las marcas recurren a los enlaces, H\&M, Ralph Lauren y Hugo Boss remiten principalmente a imágenes (46,8\%, 69,4\% y $71 \%$ de los enlaces respectivamente). Sin embargo, aunque Zara prefiere las páginas webs (47,9\%), también es habitual que utilice imágenes (45\%). Además, Zara es la única de las marcas analizadas que no redirige en ninguna ocasión a Instragram o a Spotify. A pesar de que todas las marcas utilizan los enlaces para introducir videos, únicamente H\&M y Hugo Boss recurren a la plataforma Youtube. Por su parte, Ralph Lauren y Hugo Boss son las únicas que enlazan a blogs, a Periscope y a medios de comunicación on-line (ver tabla 2).

Tabla 2. Lugar donde dirigen de los enlaces en función de la marca

\begin{tabular}{|c|c|c|c|c|c|c|}
\hline \multirow{2}{*}{$\begin{array}{l}\text { Lugar donde } \\
\text { dirige el } \\
\text { enlacea }\end{array}$} & & \multicolumn{4}{|c|}{ Marca } & \multirow[t]{2}{*}{ TOTAL } \\
\hline & & H\&M & Zara & $\begin{array}{c}\text { Ralph } \\
\text { Lauren }\end{array}$ & $\begin{array}{l}\text { Hugo } \\
\text { Boss }\end{array}$ & \\
\hline \multirow{4}{*}{ Web } & Recuento & 264 & 116 & 23 & 30 & 433 \\
\hline & \% lugar & 61 & 26,8 & 5,3 & 6,9 & \\
\hline & $\%$ marca & 43,6 & 47,9 & 8,5 & 7,6 & \\
\hline & $\%$ total & 17,5 & 7,7 & 1,5 & 2 & 28,7 \\
\hline \multirow{4}{*}{ Twitter } & Recuento & 7 & 1 & 5 & 1 & 14 \\
\hline & \% lugar & 50 & 7,1 & 35,7 & 7,1 & \\
\hline & $\%$ marca & 1,2 & 0,4 & 1,8 & 0,3 & \\
\hline & $\%$ total & 0,5 & 0,1 & 0,3 & 0,1 & 0,9 \\
\hline \multirow{4}{*}{ Youtube } & Recuento & 15 & 0 & 0 & 1 & 16 \\
\hline & \% lugar & 93,8 & 0 & 0 & 6,3 & \\
\hline & $\%$ marca & 2,5 & 0 & 0 & 0,3 & \\
\hline & $\%$ total & 1 & 0 & 0 & 0,1 & 1,1 \\
\hline \multirow{4}{*}{ Instagram } & Recuento & 4 & 0 & 7 & 3 & 14 \\
\hline & \% lugar & 28,6 & 0 & 50 & 21,4 & \\
\hline & $\%$ marca & 0,7 & 0 & 2,6 & 0,8 & \\
\hline & $\%$ total & 0,3 & 0 & 0,5 & 0,2 & 0,9 \\
\hline \multirow{4}{*}{ Periscope } & Recuento & 0 & 0 & 2 & 2 & 4 \\
\hline & \% lugar & 0 & 0 & 50 & 50 & \\
\hline & $\%$ marca & 0 & 0 & 0,7 & 0,5 & \\
\hline & $\%$ total & 0 & 0 & 0,1 & 0,1 & 0,3 \\
\hline \multirow{4}{*}{ Blog } & Recuento & 0 & 0 & 3 & 5 & 8 \\
\hline & \% lugar & 0 & 0 & 37,5 & 62,5 & \\
\hline & $\%$ marca & 0 & 0 & 1,1 & 1,3 & \\
\hline & $\%$ total & 0 & 0 & 0,2 & 0,3 & 0,5 \\
\hline
\end{tabular}




\begin{tabular}{|c|c|c|c|c|c|c|}
\hline $\begin{array}{l}\text { Medios de } \\
\text { comunicación } \\
\text { on-line }\end{array}$ & $\begin{array}{l}\text { Recuento } \\
\% \text { lugar } \\
\% \text { marca } \\
\% \text { total }\end{array}$ & $\begin{array}{l}0 \\
0 \\
0 \\
0\end{array}$ & $\begin{array}{l}0 \\
0 \\
0 \\
0\end{array}$ & $\begin{array}{c}10 \\
62,5 \\
3,7 \\
0,7\end{array}$ & $\begin{array}{c}6 \\
37,5 \\
1,5 \\
0,4\end{array}$ & 1,1 \\
\hline Imagen & $\begin{array}{l}\text { Recuento } \\
\% \text { lugar } \\
\% \text { marca } \\
\% \text { total } \\
\end{array}$ & $\begin{array}{l}283 \\
32,9 \\
46,8 \\
18,7 \\
\end{array}$ & $\begin{array}{c}109 \\
12,7 \\
45 \\
7,2\end{array}$ & $\begin{array}{l}188 \\
21,9 \\
69,4 \\
12,4 \\
\end{array}$ & $\begin{array}{c}279 \\
32,5 \\
71 \\
18,5\end{array}$ & 859 \\
\hline Videos & $\begin{array}{l}\text { Recuento } \\
\% \text { lugar } \\
\% \text { marca } \\
\% \text { total }\end{array}$ & $\begin{array}{c}29 \\
21,2 \\
4,8 \\
1,9\end{array}$ & $\begin{array}{c}16 \\
11,7 \\
6,6 \\
1,1\end{array}$ & $\begin{array}{c}32 \\
23,4 \\
11,8 \\
2,1\end{array}$ & $\begin{array}{c}60 \\
43,8 \\
15,3 \\
4\end{array}$ & 137 \\
\hline Spotify & $\begin{array}{l}\text { Recuento } \\
\% \text { lugar } \\
\% \text { marca } \\
\% \text { total }\end{array}$ & $\begin{array}{c}2 \\
66,7 \\
0,3 \\
0,1\end{array}$ & $\begin{array}{l}0 \\
0 \\
0 \\
0\end{array}$ & $\begin{array}{c}1 \\
33,3 \\
0,4 \\
0,1\end{array}$ & $\begin{array}{l}0 \\
0 \\
0 \\
0\end{array}$ & 0,2 \\
\hline No encontrado & $\begin{array}{l}\text { Recuento } \\
\% \text { lugar } \\
\% \text { marca } \\
\% \text { total }\end{array}$ & $\begin{array}{c}1 \\
14,3 \\
0,2 \\
0,1\end{array}$ & $\begin{array}{l}0 \\
0 \\
0 \\
0\end{array}$ & $\begin{array}{l}0 \\
0 \\
0 \\
0\end{array}$ & $\begin{array}{c}6 \\
85,7 \\
1,5 \\
0,4 \\
\end{array}$ & 0,5 \\
\hline Total & $\begin{array}{l}\text { Recuento } \\
\% \text { total }\end{array}$ & $\begin{array}{c}605 \\
40\end{array}$ & $\begin{array}{c}242 \\
16\end{array}$ & $\begin{array}{l}271 \\
17,9\end{array}$ & $\begin{array}{c}393 \\
26\end{array}$ & $\begin{array}{c}1511 \\
100\end{array}$ \\
\hline
\end{tabular}

Los porcentajes y los totales se basan en las respuestas.

\subsection{Principio de conservación de los visitantes}

Al examinar la frecuencia de publicación, y como ya se ha comentado, cabe destacar que H\&M es la que más tweets posee con el 35,6\% del total (404 tweets), seguido de Hugo Boss con el 30,5\% (346 tweets), de Ralph Lauren con el 22,9\% (260 tweets) y, finalmente, de Zara con el 11\% (125 tweets).

En este sentido, tampoco existe homogeneidad entre las marcas al considerar la fecha de publicación. Así, se producen diferencias estadísticamente significativas en función de los meses estudiados $\left(x^{2}=45,597 ; p=0,000\right)$. De esta forma, y como se puede observar en el gráfico 1, H\&M y Ralph Lauren realizan más publicaciones en febrero (99 y 70 tweets respectivamente), mientras que Hugo Boss prefiere marzo (73), mes en el que Zara realiza menos publicaciones (10) al decantarse por mayo (31 tweets). 


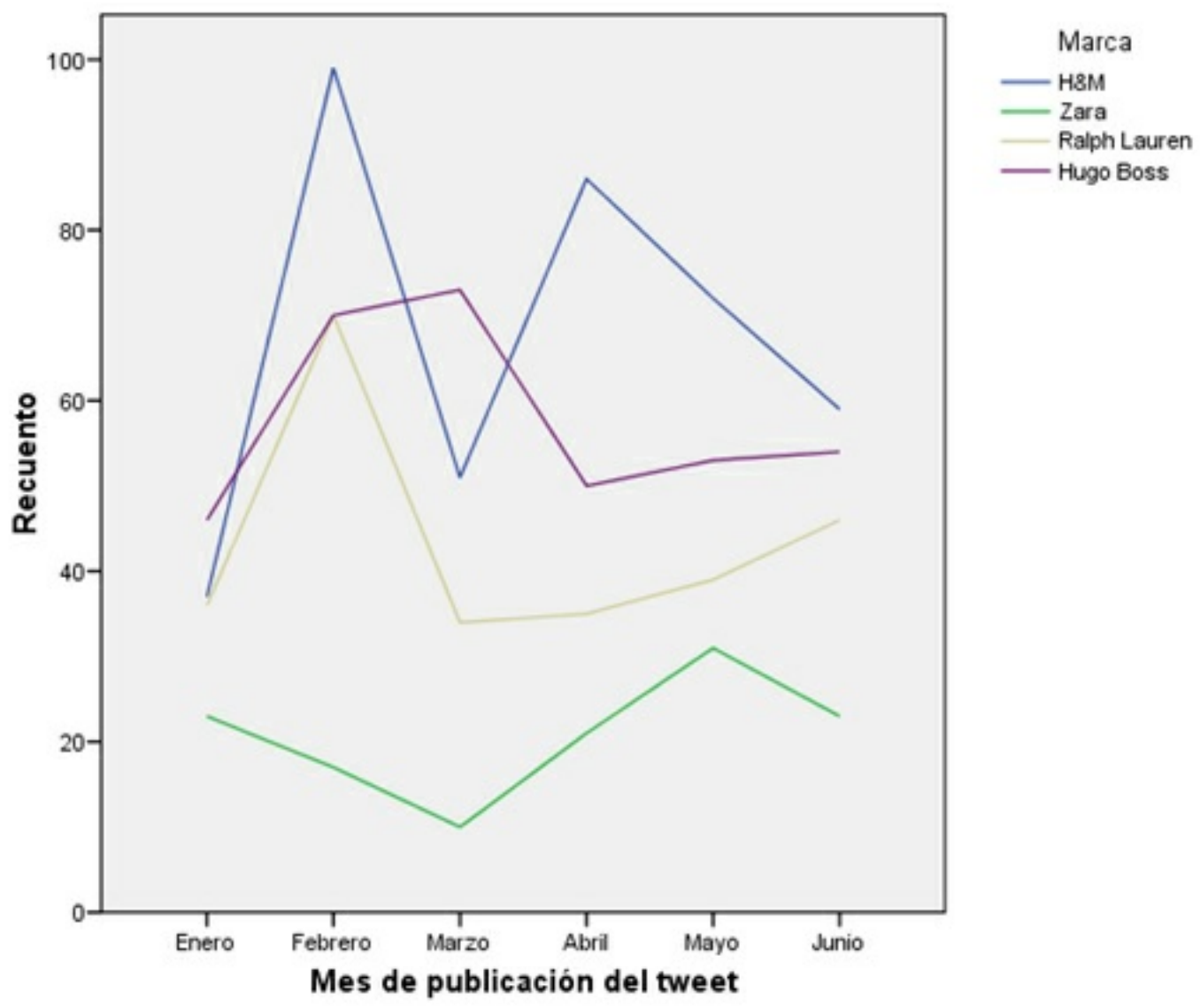

Fuente: elaboración propia a través del programa informático SPSS

Gráfico 1. Número de tweets publicados en función del mes y de la marca

También se producen desemejanzas estadísticamente significativas al analizar el día del mes más prolífico $\left(x^{2}=129,016 ; p=0,004\right)$ : H\&M y Zara prefieren publicar a principios de mes, en concreto el día 2, frente a Ralph Lauren y Hugo Boss que utilizan mayoritariamente los días centrales, específicamente la primera de estas marcas publica principalmente el día 18 y la segunda recurre a los días 17 y 18 con la misma frecuencia (ver gráfico 2). 


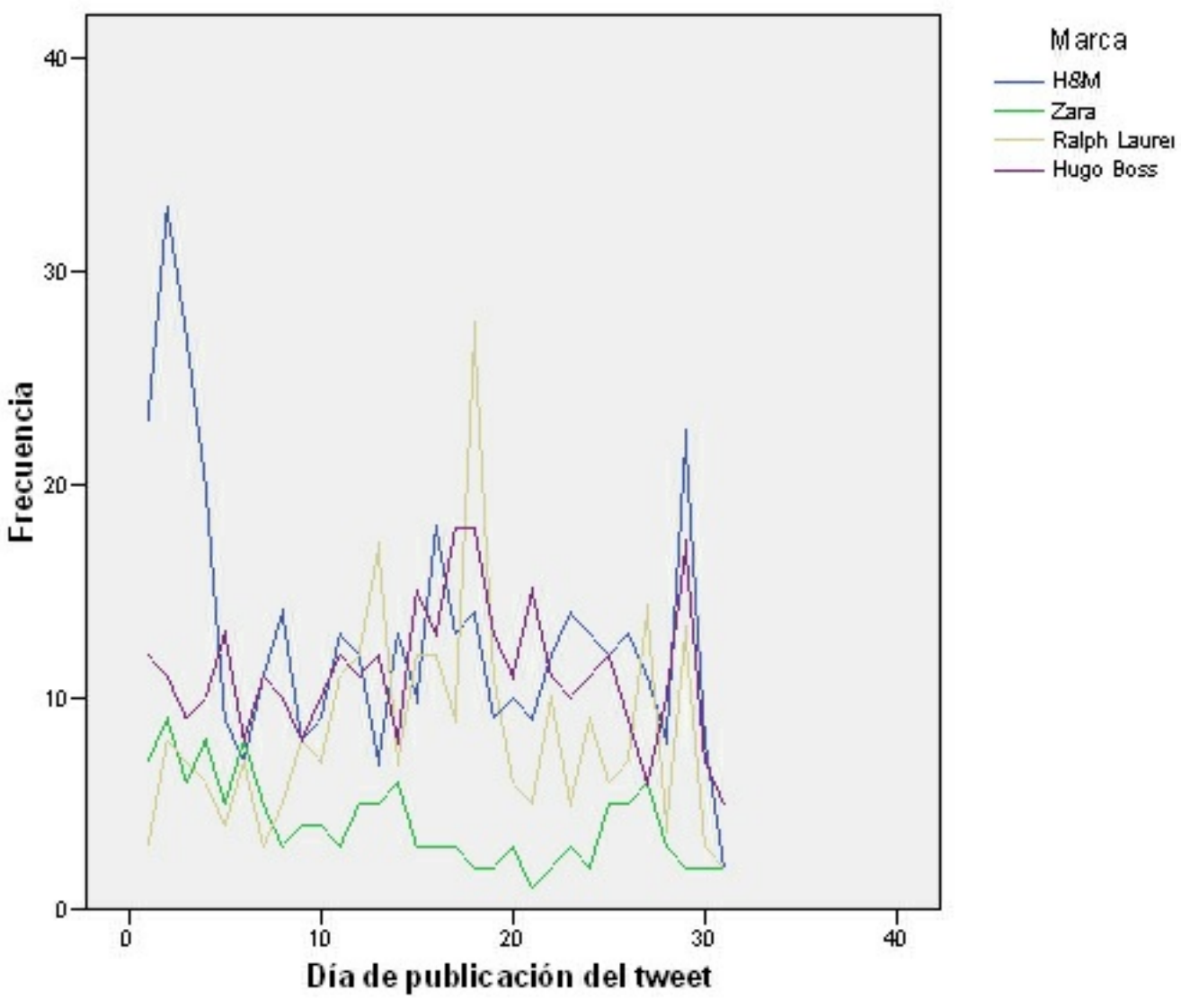

Fuente: elaboración propia a través del programa informático SPSS

Gráfico 2. Número de tweets publicados en función del día y de la marca

Al examinar si los enlaces llevan a sus seguidores a sitios relacionados con la propia marca, cabe destacar que, en general, la mayoría redirigen a lugares corporativos. De esta forma, Zara no utiliza ningún enlace externo, H\&M recurre a ellos en el 4,1\% de sus enlaces, Hugo Boss en el 6,4\% y Ralph Lauren en el 22,5\% (Gráfico 5).

\subsection{Principio de generación de visitas reiteradas}

Como se puede observar en la tabla 3, ninguna de las marcas analizadas utiliza enlaces que redirigen a sus seguidores a páginas en las que pueden solicitar información adicional, en las que haya foros de discusión o en las que se resuelvan preguntas frecuentes -Frequently Asked Questions (FAQs)-. Sin embargo, Ralph Lauren, H\&M y Hugo Boss sí incluyen enlaces con información sobre eventos de la propia marca $(16,2 \%, 13,6 \%$ y 4,3\% de los enlaces respectivamente) y con noticias en medios de comunicación (6,6\%, 0,2\% y $1 \%$ respectivamente). Aunque en escasas ocasiones, todas las marcas incorporan enlaces centrados en la venta on-line: Hugo Boss en el $7,9 \%$ de las respuestas, Ralph Lauren en el $7,4 \%$, Zara en el $5 \%$ y $\mathrm{H} \& \mathrm{M}$ en el $0,2 \%$. 
Tabla 3. Contenido de los enlaces en función de la marca

\begin{tabular}{|c|c|c|c|c|c|c|}
\hline \multirow{2}{*}{$\begin{array}{l}\text { Contenido de los } \\
\text { enlacess }\end{array}$} & & \multicolumn{4}{|c|}{ Marca } & \multirow[t]{2}{*}{ TOTAL } \\
\hline & & H\&M & Zara & $\begin{array}{l}\text { Ralph } \\
\text { Lauren }\end{array}$ & $\begin{array}{l}\text { Hugo } \\
\text { Boss }\end{array}$ & \\
\hline \multirow{4}{*}{$\begin{array}{l}\text { Información sobre } \\
\text { eventos de la } \\
\text { compañía }\end{array}$} & Recuento & 82 & 0 & 44 & 17 & 143 \\
\hline & $\%$ contenido & 57,3 & 0 & 30,8 & 11,9 & \\
\hline & $\%$ marca & 13,6 & 0 & 16,2 & 4,3 & \\
\hline & $\%$ total & $5,4 \%$ & 0 & 2,9 & 1,1 & 9,5 \\
\hline \multirow{4}{*}{$\begin{array}{l}\text { Noticias/Aparicio- } \\
\text { nes de la } \\
\text { compañía en } \\
\text { medios de } \\
\text { comunicación }\end{array}$} & Recuento & 1 & 0 & 18 & 4 & 23 \\
\hline & $\%$ contenido & 4,3 & 0 & 78,3 & 17,4 & \\
\hline & $\%$ marca & 0,2 & 0 & 6,6 & 1 & \\
\hline & $\%$ total & 0,1 & 0 & 1,2 & 0,3 & 1,5 \\
\hline \multirow{4}{*}{ Venta online } & Recuento & 1 & 12 & 20 & 31 & 64 \\
\hline & $\%$ contenido & 1,6 & 18,8 & 31,3 & 48,4 & \\
\hline & $\%$ marca & 0,2 & 5 & 7,4 & 7,9 & \\
\hline & $\%$ total & 0,1 & 0,8 & 1,3 & 2,1 & 4,2 \\
\hline \multirow{4}{*}{ Otros } & Recuento & 381 & 229 & 183 & 333 & 1126 \\
\hline & $\%$ contenido & 33,8 & 20,3 & 16,3 & 29,6 & \\
\hline & $\%$ marca & 63 & 94,6 & 67,5 & 84,7 & \\
\hline & $\%$ total & 25,2 & 15,2 & 12,1 & 22 & 74,5 \\
\hline \multirow{4}{*}{ No encontrado } & Recuento & 140 & 1 & 6 & 8 & 155 \\
\hline & $\%$ contenido & 90,3 & 0,6 & 3,9 & 5,2 & \\
\hline & $\%$ marca & 23,1 & 0,4 & 2,2 & 2,0 & \\
\hline & $\%$ total & 9,3 & 0,1 & 0,4 & 0,5 & 10,3 \\
\hline \multirow{2}{*}{ Total } & Recuento & 605 & 242 & 271 & 393 & 1511 \\
\hline & $\%$ total & 40 & 16 & 17,9 & 26 & 100 \\
\hline
\end{tabular}

Los porcentajes y los totales se basan en las respuestas.

\section{a. Agrupación}

Fuente: elaboración propia a través del programa informático SPSS

Con referencia al uso de menciones cabe señalar que las diferencias que se producen entre las marcas son estadísticamente significativas $\left(x^{2}=216,394 ; p=0,000\right)$. De esta forma, Zara no incluye ninguna mención en sus publicaciones, mientras que H\&M las utiliza en el $40,1 \%$ de sus tweets, Hugo Boss en el $41,9 \%$ y Ralph Lauren en el $60,8 \%$. En este sentido, cuando las marcas recurren a las menciones lo más habitual es que únicamente muestren una por tweet.

La mención que aparece con mayor frecuencia (4) es @Alexthomson99, un regatista profesional británico patrocinado por Hugo Boss al que se hace referencia en 28 tweets de dicha marca $(8,09 \%$ de las publicaciones de Hugo Boss). H\&M menciona en 7 publicaciones (1,73\%) a @amanda_bisk, una deportista australiana referente en el ámbito del yoga, y Ralph Lauren cita en 6 ocasiones (2,31\%) a @RalphsCoffee, una cuenta de Twitter relacionada con la marca y el café. Como ya se ha comentado, Zara no incluye ninguna mención en sus tweets.

Sin embargo, los hashtags son utilizados por todas las marcas con frecuencia. Hugo Boss recurre a ellos en el $95,1 \%$ de los tweets, seguido de Zara que los usa en el $90,4 \%$, H\&M en el $80 \%$ y Ralph Lauren en el $73,1 \%$. En este sentido, Zara y Hugo Boss se decantan por introducir dos hashtags en sus tweets $(50,4 \%$ y $40,8 \%$ de las ocasiones respectivamente), mientras que $\mathrm{H} \& \mathrm{M}$ es la única de las marcas analizadas que no introduce cuatro hashtags en ningún tweet. Estas diferencias entre marcas son estadísticamente significativas $\left(x^{2}=163,202\right.$; $\mathrm{p}=0,000)$. 
\#thisisboss es el hashtag que se repite con mayor asiduidad en los tweets analizados (5), ya que aparece en 94 publicaciones de Hugo Boss (27,17\% de los tweets de dicha marca). Por su parte, H\&M recurre a \#H\&MLife en 60 ocasiones (14,85\%), Zara se decanta por \#Zaraeditorials, pues lo cita en 18 tweets $(14,4 \%)$ y, curiosamente, Ralph Lauren utiliza con mayor frecuencia (22 casos, 8,5\%) un hashtag que no está relacionado directamente con su marca, \#NYFW. Cabe destacar que este último hashtag, \#NYFW (acrónimo de New York Fashion Week) también es utilizado por otra marca, concretamente en 4 ocasiones por Hugo Boss.

\subsection{Principio de mantenimiento del bucle dialógico}

Aunque no es habitual que las marcas planteen preguntas directas (6) en sus tweets, se producen diferencias estadísticamente significativas con referencia a esta cuestión $\left(x^{2}=106,650 ; p=0.000\right)$, ya que H\&M propone este tipo de interacción con sus seguidores en el $20,3 \%$ de las ocasiones, mientras que Hugo Boss lo hace en el $3,5 \%$, Zara en el $1,6 \%$ y Ralph Lauren en el $1,2 \%$.

A pesar de la información anterior, es habitual que los seguidores interactúen con la marca. En concreto, en el $87,2 \%$ de los tweets de Zara se pueden leer comentarios de sus seguidores, esta cifra desciende ligeramente en el caso de Ralph Lauren y de H\&M (84,2\% y 78,2\%). Sin embargo, los usuarios de Hugo Boss son considerablemente menos participativos $(53,2 \%)$, lo que conlleva que se produzcan diferencias estadísticamente significativas en función de la marca $\left(x^{2}=103,853 ; p=0,000\right)$.

El número de interacciones que los seguidores realizan también muestra diferencias estadísticamente significativas en función de la marca $\left(x^{2}=229,747 ; p=0,000\right)$. De esta forma, y como era de esperar, la media de comentarios en Hugo Boss es de 2,39, frente a los 3,08 de H\&M, los 3,11 de Zara y los 4,63 de Ralph Lauren.

Aunque no es habitual que en estos comentarios los seguidores realicen preguntas de forma directa a las marcas, las desemejanzas que se producen también son estadísticamente significativas $\left(x^{2}=65,271 ; p=0,000\right)$. Así, los seguidores de Hugo Boss vuelven a ser los menos participativos, pues únicamente se pueden observar preguntas en el $8,4 \%$ de los tweets, frente al $34,4 \%$ de Zara (a H\&M le realizan preguntas en el $24,5 \%$ de las ocasiones y a Ralph Lauren en el $11,2 \%$ ).

En este sentido, cabe señalar que la marca no suele volver a interactuar con sus seguidores a raíz de un tweet. De hecho, Ralph Lauren no realiza ningún comentario en ninguna de sus publicaciones y Hugo Boss únicamente lo hace en un tweet. Sin embargo, Zara recurre a este tipo de interacciones en el 25,6\% de los casos y H\&M en el 13,4\%. Así, estas diferencias, que suman más de 25 puntos entre Zara y Ralph Lauren, son estadísticamente significativas $\left(x^{2}=123,544 ; p=0,000\right)$.

El número de retweets que obtienen las publicaciones analizadas también muestran desemejanzas estadísticamente significativas $\left(x^{2}=1094,207 ; p=0,000\right)$. De esta forma, Ralph Lauren es la marca con más retweets, con una media de 150,37 por publicación, seguida de H\&M $(78,57)$, Hugo Boss $(55,56)$ y Zara $(47,14)$. En este sentido cabe señalar que H\&M es la única que posee publicaciones sin ningún retweet, en concreto 17, mientras que Ralph Lauren destaca por tener un tweet con 4.922 retweets (se trata a su vez de un retweet que realiza la marca sobre una publicación de Kim Kardashian en la que habla de Ralph Lauren).

Por su parte, cabe señalar que todos los tweets analizados han sido marcados como mínimo en una ocasión como favoritos. Vuelve a destacar el retweet de Ralph Lauren a Kim Kardashian mencionado anteriormente, ya que obtiene un total de 24.698 favoritos. En este sentido, y al igual que con los retweets, Ralph Lauren es la marca con más favoritos, pues posee una media de 457,41 por publicación, a continuación se sitúan H\&M con 323,86 , Zara con 173,27 y Hubo Boss con 150,44. Estas diferencias son estadísticamente significativas $\left(x^{2}=2070,923 ; p=0,000\right)$.

\section{CONCLUSIONES}

Los principales resultados del presente trabajo ponen de manifiesto que no existe consenso entre las marcas de 
moda estudiadas con relación al diálogo que establecen con sus seguidores. De hecho, las diferencias que se han encontrado entre los perfiles examinados son estadísticamente significativas en todos los casos, si bien destacan por sus particularidades las estrategias llevadas a cabo por Zara frente al resto.

Las marcas de moda no están aprovechando el potencial dialógico que ofrece Twitter para relacionarse con sus seguidores, ya que su principal actividad es la comunicación de imágenes, noticias y novedades.

A pesar de ello, las marcas analizadas, en general, parecen cumplir con el principio de información útil. En concreto, es habitual que los cuatro perfiles examinados incluyan enlaces en sus tweets, requisito esencial para poder ampliar información en una red social como Twitter.

Estos links, al ser en su mayoría corporativos, también fomentan la conservación de los visitantes.

Especialmente llamativo es el caso de Zara, pues es la única marca que posee enlaces en todos sus tweets y ninguno de ellos es externo.

Sin embargo, su frecuencia de publicación no es muy elevada, situación que podría afectar negativamente a la conservación de los visitantes, ya que únicamente ha escrito 125 tweets en 6 meses (media de 0,69 tweets diarios), frente a H\&M que cuenta con 404 (media de 2,22 tweets diarios).

Por otra parte, parece que las marcas examinadas no fomentan la generación de visitas reiteradas, ya que ninguna redirige a sus usuarios a lugares donde solicitar información adicional, a foros de discusión o a espacios donde se resuelvan preguntas frecuentes (FAQs). Además, Zara no incluye enlaces con información sobre eventos propios ni noticias relacionadas con la empresa en medios de comunicación, tampoco incorpora menciones en sus tweets.

Las marcas también parecen prestar poca atención al último de los principios analizados, el mantenimiento del bucle dialógico. De esta forma, no es habitual que los perfiles examinados planteen preguntas en sus tweets, aunque existe una gran disparidad entre las organizaciones (H\&M realiza preguntas en más del $20 \%$ de sus publicaciones mientras que Ralph Lauren únicamente recurre a ellas en el del 1,2\%). A pesar de que los seguidores comenten los tweets, no es frecuente que las instituciones respondan a estas interacciones, ni siquiera cuando lo hacen con una pregunta directa a la marca. De hecho, Ralph Lauren no responden en ninguna ocasión y Hugo Boss únicamente lo hace en un tweet. También en este punto, el comportamiento de Zara vuelve a ser sustancialmente distinto al resto, ya que contesta en el $25 \%$ aproximadamente de sus publicaciones.

Por su parte, la diferencia tan notable entre el número de seguidores y de amigos evidencia la falta de escucha por parte de las marcas, aspecto fundamental para el establecimiento de un verdadero diálogo. Así pues, las marcas dialogan poco con los usuarios y en raras ocasiones contribuyen a la comunidad amplificando el contenido de sus seguidores. Cabe destacar el caso de Zara, ya que en ningún caso retweetea mensajes ni utiliza menciones en sus tweets.

Por tanto, y a pesar de las diferencias halladas entre las marcas examinadas, se puede concluir que, aunque Twitter posee un amplio potencial dialógico, las marcas de moda lo utilizan mínimamente. Estos resultados coinciden con los obtenidos en anteriores investigaciones, ya que las organizaciones no suelen aprovechar completamente el potencial dialógico que ofrece la comunicación on-line para relacionarse con sus stakeholders, ya sea a través de sitios web (Kent, Taylor y White, 2003; Taylor, Kent y White, 2001; Park y Reber, 2008) o de redes sociales (Rybalko y Seltzer, 2010).

La principal limitación del presente estudio se basa en haber analizado solamente la comunicación que realizan las marcas de moda a través de una única plataforma (Twitter). Por tanto, estas empresas podrían, o no, estar aprovechando mejor el potencial dialógico que ofrecen las nuevas tecnologías. Esta situación abre una posible línea de investigación centrada en examinar la comunicación de las marcas de moda en otras plataformas digitales, como por ejemplo Instagram o Facebook. 


\section{REFERENCIAS BIBLIOGRÁFICAS}

ALONSO, M.: “Comunicación empresarial de las empresas del lbex 35 en Twitter". Revista Mediterránea de Comunicación, vol.7, nº 1, (2016), pp. 137-161.

ATARAMA, T.; CORTÉS, C.: "La gestión de la reputación digital en las universidades. Twitter como herramienta de la comunicación reputacional en las universidades peruanas". Revista de Comunicación, vol.14 (2015), pp. 27-47.

CÁCERES, M.D.; DÍAZ, P.: "La representación del cuerpo de la mujer en la publicidad de revistas femeninas". Estudios sobre el Mensaje Periodístico, vol.14 (2008), pp. 309-327.

CASTELLÓ, A.; ROS, V.J.: "El uso de Twitter para la comunicación de la responsabilidad". Revista Telos: Cuadernos de Comunicación e Innovación, nº 91, (2012), pp. 116-130.

CASTELLÓ, A.; DEL PINO, C.; RAMOS, I.: "Twitter como canal de comunicación corporativa y publicitaria”. Comunicación y Sociedad, vol.27, n² 2 (2014), pp. 21-54.

DÍAZ, P.: "Valores y estereotipos femeninos creados en la publicidad gráfica de las marcas de moda de lujo en España”. Anàlisi: Quaderns de Comunicació i Cultura, nº 35 (2007), pp. 27-45.

DÍAZ, P.; QUINTAS, N.; MUÑIZ, C.: "Cuerpos mediáticos versus cuerpos reales: un estudio de la representación del cuerpo femenino en la publicidad de marcas de moda en España", Icono 14, vol.8, n 3 (2010), pp. 244-256.

DÍAZ, P.; MUÑIZ, C.: "Valores y estereotipos femeninos creados en la publicidad gráfica de las marcas de moda de lujo en España". Zer: Revista de Estudios de Comunicación, vol. 12, nº 23 (2007), pp. 75-94.

DÍAZ, P.; MUÑIZ, C.; QUINTAS, N. “Un análisis longitudinal de 2002 a 2008. La imagen femenina en la publicidad gráfica de moda”. Revista Telos: Cuadernos de Comunicación e Innovación, nº 84 (2010), pp. 122133.

GUZMÁN, A.P.; DEL MORAL, M.E.; GONZÁLEZ, F.; GIL, H.: "Impacto de Twitter en la comunicación y promoción institucional de las universidades”. Píxel-Bit. Revista de Medios y Educación, n 43 (2013), pp. 139153.

KENT, M.L.; TAYLOR, M.; WHITE, W.J.: "The relationship between web site design and organizational responsiveness to stakeholders". Public Relations Review, vol. 29, nº 1 (2003), pp. 63-77.

KENT, M.L.; TAYLOR, M.: "Building dialogic relationships through the World Wide Web". Public Relations Review, vol. 24, n 3 (1998), pp. 321-334.

MANIKONDA, L.; VENKATESAN, R.; KAMBHAMPATI, S.; LI, B. (2014): Trending chic: analyzing the influence of social media on fashion brands [Consulta: 28 octubre 2016]. <http://arxiv.org/pdf/1512.01174.pdf>.

MARTÍNEZ, J.; MOREJÓN, N.; RAMOS D.: El emprendimiento femenino en Internet: los blogs de moda y belleza como creadores de tendencias y generadores de negocio y riqueza. En CARO, F.J. (dir.); NOGALES, A.I. (coord.) (2014): Emprender en femenino en la comunicación. La Laguna (Tenerife): Sociedad Latina de Comunicación Social, pp. 115-142.

PARK, H.; REBER, B.: "Relationship building and the use of Websites: how Fortune 500 companies use their Websites to build relationships". Public Relations Review, vol.34, nº 4 (2008), pp. 409-411.

RIERA, S.; FIGUERAS, M.: "El modelo de belleza de la mujer en los blogs de moda. ¿Una alternativa a la prensa femenina tradicional?". Cuestiones de Género, nº 7 (2012), pp. 157-176.

RYBALKO, S.; SELTZER, T.: "Dialogic communication in 140 characters or less: how Fortune 500 companies engage stakeholders using Twitter". Public Relations Review, vol.36, nº 4 (2010), pp. 336-341. 
SHARMA, R. B.; SAHNI, M.M.: "Evaluating the efficacy of facebook communities \& Twitter tweets on brand equity: an empirical study on fashion brands". Advances in Economics and Business Management (AEBM), vol.2, $n^{\circ} 5$ (2015), pp. 503-508.

SIMELIO, N.; MOLINA, P.: "Comunicación pública y participación ciudadana. El uso de Twitter en los ayuntamientos de Cataluña". Historia y Comunicación Social, vol.19 (2014), pp. 479-490.

TAYLOR, M.; KENT, M.L.; WHITE, W.J.: "How activist organizations are using the internet to build relationships". Public Relations Review, vol.27, nº 3 (2001), pp. 263-284.

VILLAFAÑE, J. (2004): La buena reputación. Claves del valor intangible de las empresas. Madrid: Ediciones Pirámide.

VILLANUEVA, J.; ACED, C.; ARMELINI, G. (2007): Los blogs corporativos: una opción, no una obligación. Cuadernos del eb Center. [Consulta: 28 octubre 2016]. <http://www.iese.edu/en/files/6_32403.pdf>.

WIMMER, R.D.; DOMINICK, J.R. (1996): La investigación científica de los medios de comunicación . Barcelona: Bosch casa editorial.

(1) Para más información véase: http://interbrand.com/best-brands/best-global-brands/2015/

(2) A pesar de que este índice ha sido calculado para todas las variables (47), en 7 ocasiones no se ha podido calcular ninguna medida de asociación por tratarse de una constante.

(3) Consideramos que las diferencias son estadísticamente significativas cuando $p<0,05$

(4) En caso de que haya más de una mención en el tweet se analiza la primera.

(5) En caso de que haya más de un hashtag en el tweet se analiza el primero.

(6) Según el protocolo utilizado para el análisis de los tweets, se ha considerado que el mensaje planteaba una pregunta en el caso de que se utilizaran los signos de interrogación, sin entrar a valorar si la marca esperaba una respuesta concreta o no.

\section{BREVE SEMBLANZA DE LAS AUTORAS}

Marián Navarro-Beltrá: Licenciada en Publicidad y RRPP (2008) por la Universidad de Alicante y Doctora Cum Laude (2013) por la misma universidad. Actualmente es profesora e investigadora en la Universidad Católica de Murcia (UCAM) en el área de Publicidad y RRPP. Sus líneas de investigación principales incluyen temas relacionados con la publicidad, las RRPP, el género y las nuevas tecnologías.

Susana Miguel-Segarra: licenciada en Ciencias de la Información, especialidad Publicidad y RRPP por la Universidad CEU San Pablo y Doctora cum Laude por la Universidad de Alicante con mención internacional (acreditado por la Glasgow Caledonian University). Actualmente es profesora en la Universitat Jaume I e investigadora en el grupo ENCOM de esta universidad, así como colaboradora en el grupo de investigación cosoco de la Universidad de Alicante.

Irene García Medina: Profesora de marketing en la Glasgow Caledonian University (Reino Unido). Sus áreas de interés en investigación son: el marketing digital, la comunicación digital y los medios de comunicación social. Licenciada en Ciencias de la Comunicación (Universidad Complutense de Madrid, España) y Doctora en 
Marketing (University of Sophia - Antipolis, France) y Relaciones Internacionales (University of Vienna, Austria).

Ámbitos. Revista Internacional de Comunicación, n.35, edición de invierno, 2016-2017.

Recibido: 22/11/2016

Aprobado: 10/12/2016 